\title{
A Recent Genetic and Antioxidant Functions of Vitamin PABA: A Review
}

\author{
Svetlana Vasilieva*1 and Anna Rylskaya ${ }^{2}$ \\ ${ }^{1}$ NM Emanuel Institute of Biochemical Physics, Russian Academy of Sciences, Russia \\ ${ }^{2} D$ Mendeleev University of Chemical Technology of Russia (student), Russia
}

Received: 眥: September 17, 2018; Published: 制 September 26, 2018

*Corresponding author: Svetlana Vasilieva, Professor, Dr. Sci, N.M. Emanuel Institute of Biochemical Physics, 4 Kosygin Street, Moscow, 119334, Russia

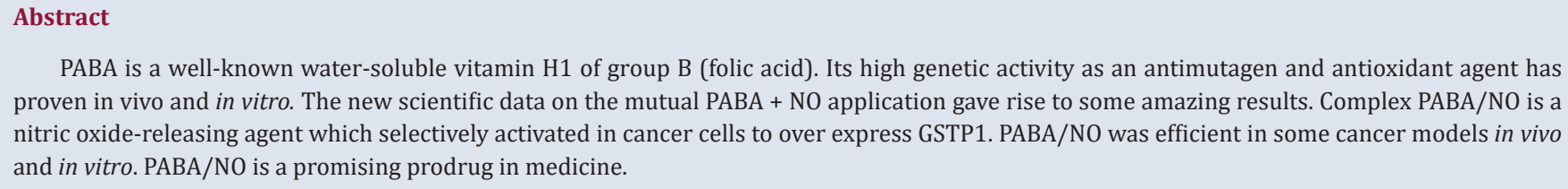

PABA is a well-known water-soluble vitamin $\mathrm{H} 1$ of group B (folic acid). Its high genetic activity as an antimutagen and antioxidant agent has proven in vivo and in vitro. The new scientific data on the mutual PABA + NO application gave rise to some amazing results. Complex PABA/NO is a nitric oxide-releasing agent which selectively activated in cancer cells to over express GSTP1. PABA/NO was efficient in some cancer models in vivo and in vitro. $\mathrm{PABA} / \mathrm{NO}$ is a promising prodrug in medicine.

Keywords: Para-aminobenzoic acid; PABA/NO; Antioxidant functions; The DNA repair SOS processes inhibitor

\section{Introduction}

Para-aminobenzoic acid (PABA) - Vitamin H1 of group B and a key cofactor for the folic acid production in bacteria [1] has obtained fundamental and practical interest as a unique watersoluble natural antimutagen since 1979 [2-4]. A priority data obtained by Russian geneticist Josef Rapoport in the 1950-th have revealed the morphogenic effects of PABA in Drosophila. Then investigations of the different test objects led to the detailed research of the amazing PABA activities. Firstly, in the experiments with the potent chemical mutagens - alkylating agents, UV- and gamma irradiation S Vasilieva et al. [5] have revealed that PABA inhibited a set of the SOS DNA repair functions in Escherichia coli cells and studied the mechanisms of the phenomenon. Mutagenic efficiency of the chemical and UV agents suppressed when PABA was added to the bacterial suspension concurrently with or prior to the mutagens. UV-spectrophotometric and NMR spectrometric measurements did not support an interaction between the direct acting chemical mutagens and PABA.

PABA modulates the accuracy of DNA repair processing by inducing the unique inducible error-prone - the SOS DNA repair pathway and decreasing the rate of mutations in E. coli cells. It inhibited the mutagenic phage lambda lysogenic induction more than two orders of magnitude, as well as its thermal shift from the permissive to non-permissive temperature in E. coli tif-1 mutant and decreased W-reactivation of UV-damaged phage lambda [6-7]. Chloramphenicol treatment of the cells just after the mutagenic treatment prevented the occurrence of PABA specific activity. These experimental results were the evidence that PABA suppressed the error-prone SOS DNA repair pathway in E. coli cells and appears to be an effective bio antimutagen [8]. To date a new stage in a PABA history has begun- its interference with low-intensive UVA (320-380nm) and UVB (280-320nm) UV radiation - as a source of ROS. The scientific data of human protection against UVA-induced biological damage is very limited. UVA caused damages may involve generation of ROS due to photosensitizing of cellular structures and inhibition of cellular antioxidants [9-10].

UVA can also damage DNA leading to single-strand breaks and inhibits DNA repair pathways [11]. UVB-absorbing sunscreen protects DNA against photocarcinogenic damages. On the model of UVA- potentiating DNA damages by Fenton reaction system PABA effects were investigated. It was demonstrated that PABA inhibited the damages in dose-dependent manner and the effect was connected with its radical-scavenging potency because PABA does not absorb in the UVA region. So, PABA is useful against photocarcinogenesis [10]. Our results with the NO-donors are consistent with the conclusion of $\mathrm{Hu}$ et al [12] that PABA strongly absorbs ROS radicals ( $\mathrm{OH}-$, singlet oxygen and HOCL), and protects DNA from the genetically dangerous UVC $(254 \mathrm{~nm})$ radiation. In our experiments in vitro PABA changed the kinetics of NO-molecules releasing out of the NO-donor solutions by increasing their stability. PABA 2,5-3 fold inhibited the DNA DSB in the mice blood leukocytes, induced by NO, and decreased the level of the bacterial biofilm formation in the stress conditions. Complex treatment of Jurkat 
cells of human T lymphocytes with NO-agent and PABA decreased the inducible DNA SSB 3-8-fold. Now vitamin PABA became a new very promising component for cosmetic production [13].

The new chemical complexes of nitric oxide (NO) and PABA have displayed a lot of surprises. Leukemia cells are very sensitive to NO and NO-donating agents which exhibited high anticancer activities against leukemia cells in vivo and in vitro [14-16]. The chemical structure of PABA/NO, (see [16-18] was produced to be activated in cancer cells, containing the abundance of glutathione S-transferase P1 (GSNP1). The mechanism of activation with a nucleophile GSH led to the formation the Meisenheimer complex that spontaneously gives two molar equivalents of nitric oxide - NO. Oxidation of the agents by ROS/RNS significantly changes the redox potency in cancer cells and triggers a complex of pro-death cellular pathways. Now PABA/NO has shown a high anticancer potency in preclinical studies. The efficacy of PABA/NO as a powerful in vivo signaling mediator was demonstrated [17-19]. On the base of our new experimental data in $E$. coli cells we have made a preliminary prognosis that vitamin PABA may be reasonable for detailed investigation in the experimental medicine as a preventive agent in hemorrhage/resuscitation-induced injuries.

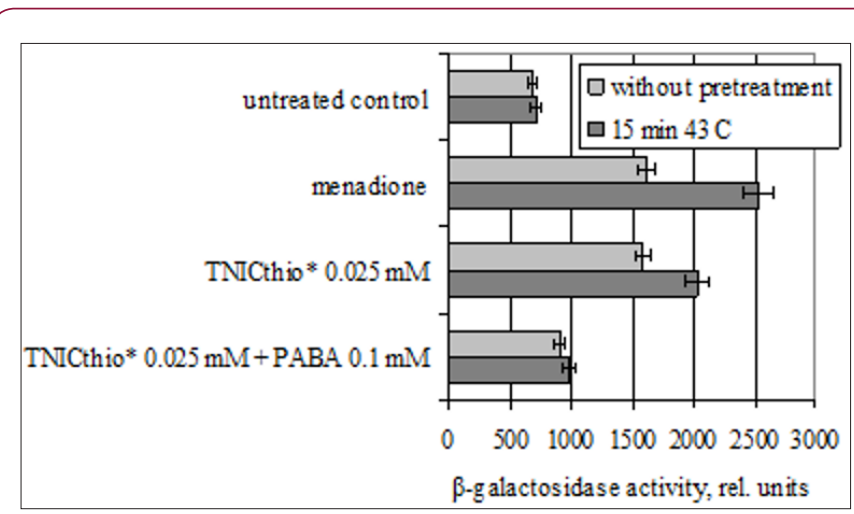

Figure 1: In E. coli cells the heat shock proteins increased the soxRS DNA repair pathway activation by NO-donor TNICthio* and $\mathrm{O}_{2}$ - generated menadione, while PABA efficiently inhibited the DNA repair response to oxidative damages, induced by the both agents.

The underlying mechanisms of the phenomenon are not studied in detail, but it has been shown that NO overproduction due to iNOS overexpression (or NO-donor application) as well as an induction of heat shock protein $70 \mathrm{kD}$ (HSP-70i) play the key roles in complex damages connected with the phenomenon. We have observed that PABA regulates both processes in the bacterial cell and strongly inhibits NO-donating processes in vitro (Figures 1 \& 2), while HSP$70 \mathrm{i}$ is common for all biosystems studied. PABA is a cofactor and precursor in the synthesis of folic acid and thymine in the bacteria, algae and higher plants. Do not forget that the lowering of the ATP level is one of the hallmarks of hemorrhage.

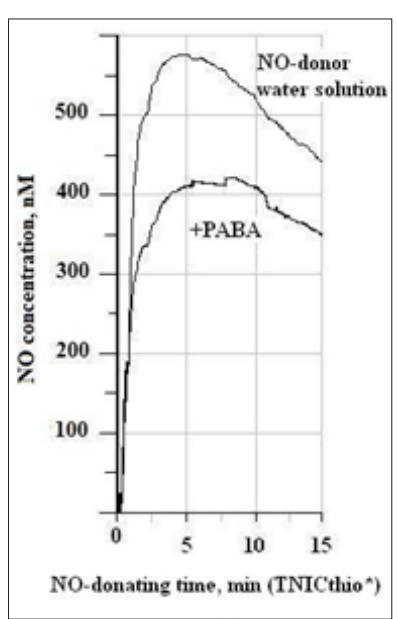

Figure 2: PABA addition to NO donor solution strongly decreased the amount of NO-molecule donating by TNICthio.

*TNICthio - tetranitrosyl iron complex with thiosulfate was synthesized at the Institute of Problems of Chemical Physics RAS by Dr N. Sanina.

\section{References}

1. Andreeva NA (1963) Vitamins of folic acid group. Akademia nauk SSSR, Russia.

2. Vasileva SV, Davnichenko LS, Lutskova EV, Rapoport IA (1979) Reparative effect of the genetically active natural compound $\mathrm{p}$-aminobenzoic acid in an experiment with N-nitrosomethylurea. Doklady Akademii nauk SSSR, 247(1): 226-230.

3. Vasileva SV, Davnichenko LS, Rapoport IA (1982) Para-aminobenzoic acid intensification of DNA repair processes in Escherichia coli K-12. Genetika 18(3): 381-391.

4. Vasilieva SV (1982) Water soluble vitamins in DNA repair processes. In Proceed of the 4th Intern. Symp on Genetics of Industrial Microorg, Japan.

5. Vasilieva SV, Rapoport IA, Davnichenko LS (1979) A role of paraaminobenzoic acid in repair of DNA damages induced by UV- and gamma radiations. Doklady Akademii nauk SSSR 247(1): 231-234.

6. Vasileva SV, Gorb TE, Rapoport IA (1983) Vitamin para-aminobenzoic acid inhibits development of SOS function in tif- 1 mutants of Escherichia coli at nonpermissive temperatures. Genetika 19(12): 1952-1957.

7. Vasileva SV, Tonkal TE (1983) para-Aminobenzoic acid inhibits the manifestation of inducible SOS functions in Escherichia coli K-12. Genetika 19(11): 1778-1785.

8. Gichner T, Velemínský J (1988) Inhibitory effects of para-aminobenzoic acid on the formation and mutagenicity of $\mathrm{N}$-nitroso compounds. Mutagenesis 3(4): 329-331.

9. Lautier D, Luscher P, Tyrrell RM (1992) Endogenous glutathione levels modulate both constitutive and UVA radiation/hydrogen peroxide inducible expression of the human heme oxygenase gene. Carcinogenesis 13(2): 227-232.

10. Shih MK, Hu ML (1996) UVA-potentiated damage to calf thymus DNA by Fenton reaction system and protection by para-aminobenzoic acid. Photochemistry and photobiology 63(3): 286-291. 
11. Sidjanin D, Zigman S, Reddan J (1993) DNA damage and repair in rabbit lens epithelial cells following UVA radiation. Current eye research 12(9) 773-781.

12. Hu ML, Chen YK, Chen LC, Sano M (1995) Para-aminobenzoic acid scavenges reactive oxygen species and protects DNA against UV and free radical damage. The Journal of Nutritional Biochemistry 6(9): 504-508.

13. Vasilieva SV, Petrishcheva MS, Gusarova EI, Osipov AN (2016) Vitamin Para-Aminobenzoic Acid (PABA) Controls Generation of Nitric Oxide (NO) In Vitro and Its Biological Functions in the Bacterial Cells. Adv Tech Biol Med 4(4): 195-200.

14. Shami PJ, Sauls DL, Weinberg JB (1998) Schedule and concentrationdependent induction of apoptosis in leukemia cells by nitric oxide. Leukemia 12(9): 1461

15. Saavedra JE, Shami PJ, Wang LY, Davies KM, Booth MN, et al. (2000) Esterase-sensitive nitric oxide donors of the diazeniumdiolate family: in vitro antileukemic activity. Journal of medicinal chemistry 43(2): 261269.

ISSN: 2574-1241

DOI: 10.26717/BJSTR.2018.09.001791

Svetlana Vasilieva. Biomed J Sci \& Tech Res

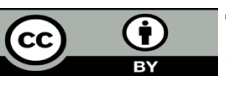

This work is licensed under Creative Commons Attribution 4.0 License

Submission Link: https://biomedres.us/submit-manuscript.php
16. Shami PJ, Saavedra JE, Wang LY, Bonifant CL, Diwan BA, et al. (2003) JS$\mathrm{K}$, a Glutathione/Glutathione S-Transferase-activated Nitric Oxide Donor of the Diazeniumdiolate Class with Potent Antineoplastic Activity1. Molecular cancer therapeutics 2(4): 409-417.

17. Saavedra JE, Srinivasan A, Buzard GS, Davies KM, Waterhouse DJ, et al. (2006) PABA/NO as an anticancer lead: analogue synthesis, structure revision, solution chemistry, reactivity toward glutathione, and in vitro activity. Journal of medicinal chemistry 49(3): 1157-1164.

18. Findlay VJ, Townsend DM, Saavedra JE, Buzard GS, Citro ML, et al. (2004) Tumor cell responses to a novel glutathione S-transferase-activated nitric oxide-releasing prodrug. Molecular pharmacology 65(5): 10701079.

19. Kim Y, Maciag AE, Cao Z, Deschamps JR, Saavedra JE, et al. (2015) PABA/ NO lead optimization: Improved targeting of cytotoxicity to glutathione S-transferase P1-overexpressing cancer cells. Bioorganic \& medicinal chemistry 23(15): 4980-4988.

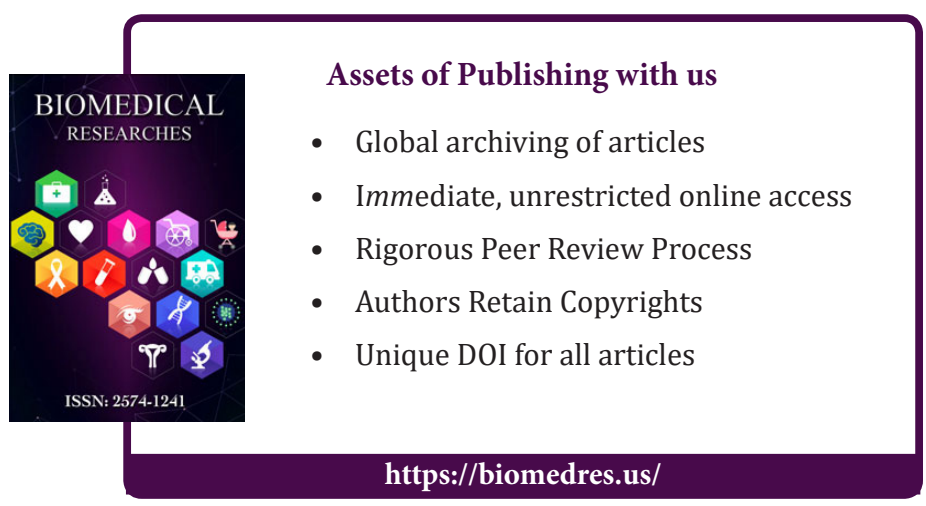

